\title{
ELABORAÇÃO DE UM INSTRUMENTO DE SISTEMATIZAÇÃO DA ASSISTÊNCIA DE ENFERMAGEM: RELATO DE EXPERIÊNCIA
}

\section{ELABORATION OF A NURSING ASSISTANCE SYSTEMATIZATION INSTRUMENT: EXPERIENCE REPORT \\ ELABORACIÓN DE UN INSTRUMENTO DE SISTEMATIZACIÓN DE LA ASISTENCIA DE ENFERMERÍA: RELATO DE EXPERIENCIA}

Fernanda Maryneve Menezes Tavares ${ }^{1}$, Walter de Souza Tavares ${ }^{2}$

\section{RESUMO}

Objetivo: descrever a experiência do enfermeiro residente de enfermagem cirúrgica na elaboração de um instrumento de sistematização da assistência de enfermagem baseado na teoria das necessidades humanas básicas de Wanda Aguiar Horta. Métodos: trata-se de uma pesquisa descritiva, exploratória com abordagem qualitativa, do tipo relato de experiência realizada em hospital púbico. Resultados: criação de um instrumento que contempla todas as fases do processo de enfermagem baseado na teoria das necessidades humanas básicas, onde a assistência de enfermagem seria sistematizada e documentada e, assim, arquivada no prontuário do paciente. Conclusão: o instrumento elaborado é uma importante estratégia para a organização das ações e operacionalização do processo de enfermagem.

Descritores: Assistência ao paciente; Teoria de enfermagem; Processo de enfermagem.

\section{ABSTRACT}

Objective: to describe resident nurse experience of surgical nursing in the elaboration of a nursing assistance systematization instrument based on Wanda Aguiar Horta's basic human needs theory. Methodology: This is a descriptive, exploratory research with a qualitative approach, of the type of experience report performed in a public hospital. Results: creation of an instrument that contemplates all phases of the nursing process based on basic human needs the theory, where nursing care would be systematized and documented and thus, stored in the patient's chart. Conclusion: the instrument developed is an important strategy for the actions organization and the nursing process operationalization.

Descriptors: Patient care; Nursing theory; Nursing process.

\section{RESUMEN}

Objetivo: describir la experiencia del enfermero residente de enfermería quirúrgica en la elaboración de un instrumento de sistematización de la asistencia de enfermería basado en la teoría de las necesidades humanas básicas de Wanda Aguiar Horta. Metodología: Se trata de una investigación descriptiva exploratoria con enfoque cualitativo, del tipo de informe de experiencia realizado en un hospital público. Resultados: creación de un instrumento que contemple todas las fases del proceso de enfermería basado en la teoría de las necesidades humanas básicas, donde la atención de enfermería sería sistematizada y documentada y, por lo tanto, almacenada en el cuadro del paciente. Conclusión: el instrumento desarrollado es una estrategia importante para la organización de acciones y operacionalización del proceso de enfermería.

Descriptores: Atención al paciente; Teoría de enfermería; Proceso de enfermería.

${ }^{1}$ Graduada em Enfermagem. Residente em Enfermagem Cirúrgica pelo Programa de Residência em Enfermagem da Secretaria de Saúde do Amapá. ${ }^{2}$ Graduado em Enfermagem. Mestre em Ciências Farmacêuticas pela Universidade Federal do Amapá. Docente na Universidade Federal do Amapá. DOI: 


\section{INTRODUÇÃO}

A Sistematização da Assistência de Enfermagem (SAE) é o método científico de trabalho que proporciona melhora significativa da qualidade da assistência prestada ao cliente através do planejamento individualizado das ações elaboradas pelo profissional enfermeiro. A SAE constitui o Processo de Enfermagem (PE) que se desenvolve com a elaboração de cinco etapas: Histórico do cliente, diagnósticos de enfermagem, planejamento de ações, implementação do plano de cuidados proposto e avaliação. Com isso, a SAE potencializa-se pelo conhecimento teórico do enfermeiro que irá realizar essas etapas e seu comprometimento com a assistência a ser prestada, a fim de obter uma visão integral, contínua e documentada que possibilita melhorias na qualidade da assistência de enfermagem ${ }^{(1-3)}$.

A Resolução 358/2009 ${ }^{(4)}$, do Conselho Federal de Enfermagem (COFEN) dispõe sobre a Sistematização da Assistência e a implementação do Processo de Enfermagem em ambientes públicos ou privados, em que ocorre o cuidado profissional de Enfermagem e oferece outras providências. Em seu artigo 4으, prescreve a função do enfermeiro cuja liderança, na execução e avaliação do Processo de Enfermagem (PE), segue o princípio da função.

Toda a constituição da SAE é privativa do enfermeiro. $O$ diagnóstico de enfermagem pode ser considerado o eixo norteador da sistematização. Responde pela identificação do estado de saúde/doença com um julgamento clínico sobre respostas potenciais da pessoa, família ou comunidade, aos problemas de saúde, proporcionando embasamento para elaborar as intervenções de enfermagem de forma a alcançar resultados pelos quais o enfermeiro é responsável ${ }^{(5)}$, o que facilita o cuidado da enfermagem.

Para melhor implementar, à SAE é orientado o uso de uma teoria de enfermagem, cuja a finalidade é descrever, explicar, diagnosticar e/ou prescrever cuidado de enfermagem, sustentando a qualidade da assistência. Desta forma, a teoria de enfermagem aponta versões de uma realidade e oferece elementos para soluções dos problemas relacionados ao fazer profissional.

No entanto, o enfermeiro tem enfrentado dificuldades na implementação da SAE como instrumento científico de trabalho. De acordo com a literatura, essas dificuldades estão diretamente relacionadas às precárias condições de trabalho, ao quantitativo insuficiente de funcionários e à formação acadêmica dos enfermeiros que não estimula a valorização da aplicabilidade da $S A E^{(6-7)}$.

Assim, este artigo teve como objetivo descrever a experiência do enfermeiro residente de enfermagem cirúrgica na elaboração de um instrumento de sistematização da assistência de enfermagem (SAE) baseado na Teoria das Necessidades Humanas Básicas (NHB) de Wanda Aguiar Horta, a qual foi escolhida por compreender a relação entre as NHB, no qual o enfermeiro pode avaliar a hierarquia das necessidades que influenciam o comportamento humano ${ }^{(8)}$, planejando e intervindo com ações que possam melhorar e/ou reestabelecer a NHB afetada.

\section{MÉTODOS}

Trata-se de uma pesquisa descritiva com abordagem qualitativa, do tipo relato de experiência, realizada em Hospital Público na cidade de Macapá-AP, no mês de janeiro de 2017. $O$ relato foi baseado na experiência do enfermeiro residente durante o Módulo de Sistematização da Assistência de Enfermagem ofertado na Residência de Enfermagem, no qual foi elaborado pela residente um instrumento para aplicação da SAE no setor da Clínica Cirúrgica do referido hospital, a partir da busca de instrumentos já utilizados em outros hospitais.

Definiu-se a clínica cirúrgica como setor para elaboração do estudo por ser um dos campos de rodízios durante a residência em Enfermagem cirúrgica. Partindo desse ponto, já havia conhecimento prévio do instrumento utilizado no referido setor e observou-se, durante a disciplina de SAE, a necessidade de adequar o instrumento que utilizavam.

O trabalho foi dividido em quatro etapas. $\mathrm{Na}$ primeira etapa, foi definida a teoria que melhor se adequava ao trabalho proposto. $\mathrm{Na}$ 
segunda etapa, realizou-se análise dos prontuários a fim de elencar as principais problemáticas nas prescrições de enfermagem baseadas no material já existente na clínica cirúrgica do hospital, sendo selecionado o que precisaria ser adequado para a elaboração do instrumento. Na terceira etapa, foi elaborado o instrumento a ser utilizado para a implementação da SAE associado às principais problemáticas identificadas anteriormente nas prescrições. $\mathrm{Na}$ quarta etapa, houve a apresentação do instrumento aos enfermeiros residentes durante o módulo supramencionado, utilizando como base, instrumentos utilizados em hospitais de referências e adequados à Teoria e realidade existente no local, ressaltando a importância da SAE e a proposta de introduzir na rotina da referida clínica à utilização do impresso.

\section{RESULTADOS E DISCUSSÃO}

A elaboração do instrumento para a aplicação da SAE no hospital em questão contemplou todas as fases do processo de enfermagem baseado na Teoria das Necessidades Humanas Básicas de Wanda de Aguiar Horta, onde a assistência de enfermagem seria sistematizada, documentada e assim, arquivada no prontuário do paciente.

A Resolução COFEN 358/2009(4) rege que a SAE deve ocorrer em toda instituição de saúde, pública e privada, sendo registrada formalmente no prontuário do paciente/cliente/usuário.

Assim, foi confeccionado um instrumento constituído de três partes: a primeira contempla o Histórico de Enfermagem e Exame Físico; a segunda os Diagnósticos de Enfermagem; e a terceira contempla a Prescrição de Enfermagem e Evolução a fim de auxiliar o enfermeiro da clínica cirúrgica na sistematização de sua assistência ao paciente.

A primeira parte do instrumento é o Histórico de Enfermagem e Exame Físico que é composto por: identificação, entrevista, exame físico e exames laboratoriais importantes, como mostra a Figura 1.

Figura 1: Histórico de Enfermagem e Exame Físico.

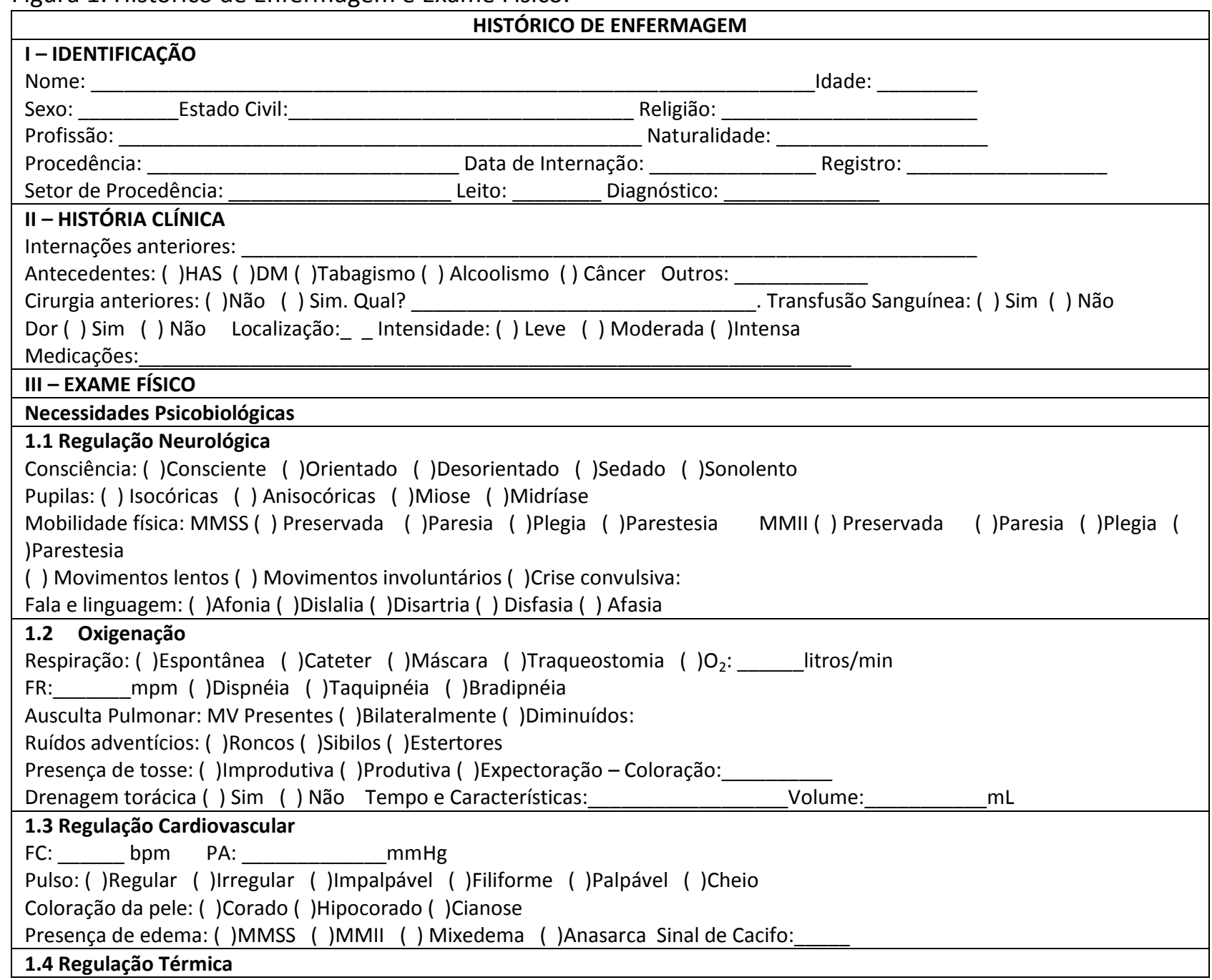




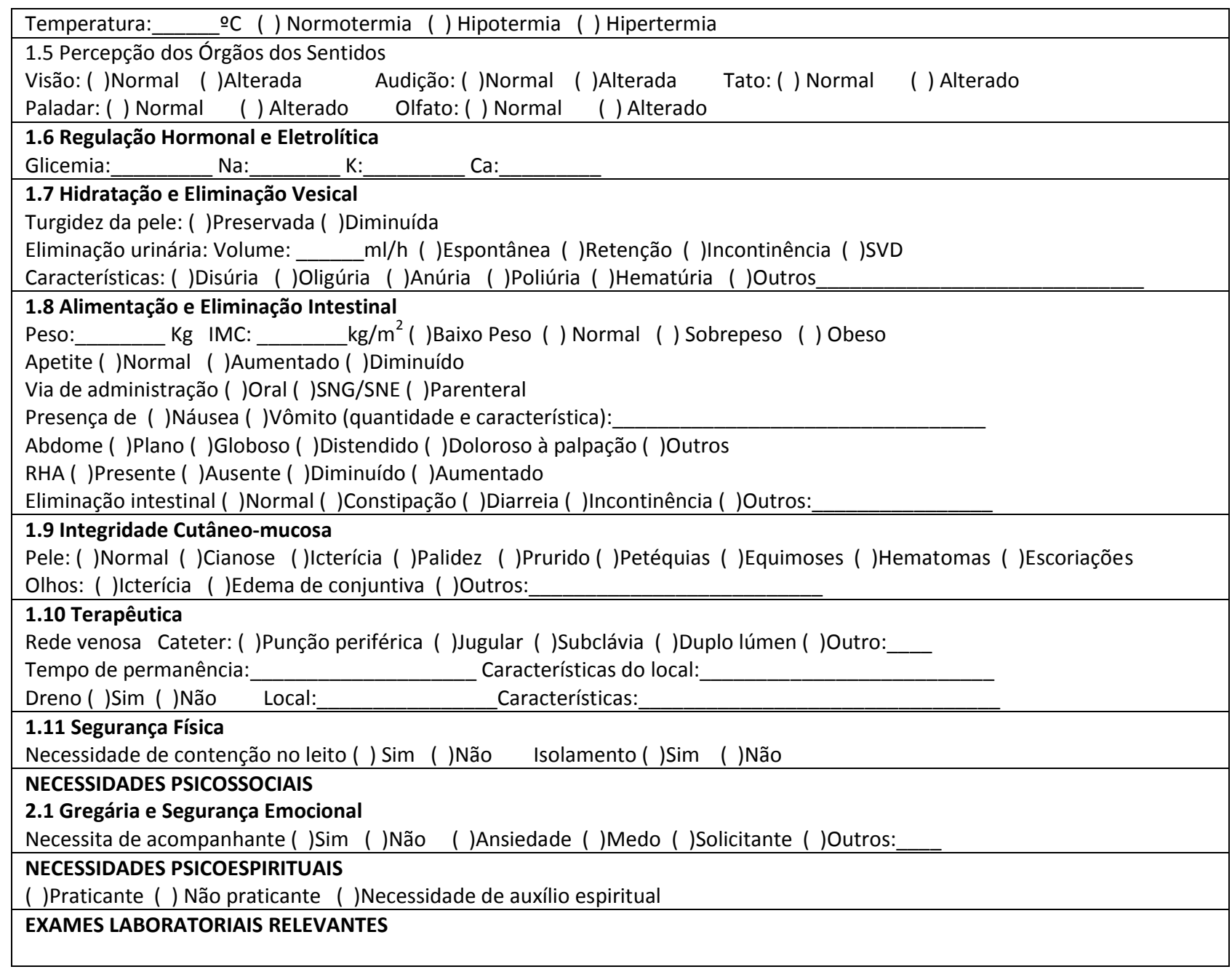

O exame físico do instrumento está subsidiado nas NHB que a Teoria de Wanda Aguiar Horta elenca, cujo objetivo é identificar as possíveis NHB afetadas no paciente.

O histórico de enfermagem, também conhecido por levantamento e investigação de dados, é um roteiro sistematizado de coleta de dados no qual se pode identificar possíveis problemas, ou seja, é a investigação das condições do paciente, tendo por finalidade conhecer os hábitos individuais e biopsicossociais, visando a identificação de problemas reais e/ou potenciais $^{(9)}$.

A coleta de dados precisos e fidedignos é imprescindível para a identificação dos problemas reais ou potenciais do paciente, mediante os quais se pode construir inferências para subsídios, a identificação dos diagnósticos de enfermagem e o direcionamento das demais etapas do processo de enfermagem ${ }^{(10)}$.

o diagnóstico de enfermagem é considerado a etapa mais complexa do processo de enfermagem, constituindo-se em importante desafio para o enfermeiro por requerer dele o pensamento crítico e conhecimentos técnicocientíficos para interpretação dos dados obtidos no exame físico e nas informações coletadas durante a anamnese ${ }^{(11)}$.

Desta forma, a segunda parte do instrumento são os Diagnósticos de enfermagem que foram elaborados de acordo com Teoria das NHB, conforme a Figura 2.

Figura 2 - Diagnóstico de enfermagem, segundo Teoria da NHB.

\begin{tabular}{|l|}
\hline Nome: \\
\hline 1 NECESSIDADES PSICOBIOLÓGICAS \\
\hline 1.1 - REGULAÇÃO NEUROLÓGICA \\
1 - Alteração no Processo de pensamento ( ) \\
- Memória prejudicada ( ) \\
3 - Confusão aguda ( ) \\
\hline
\end{tabular}




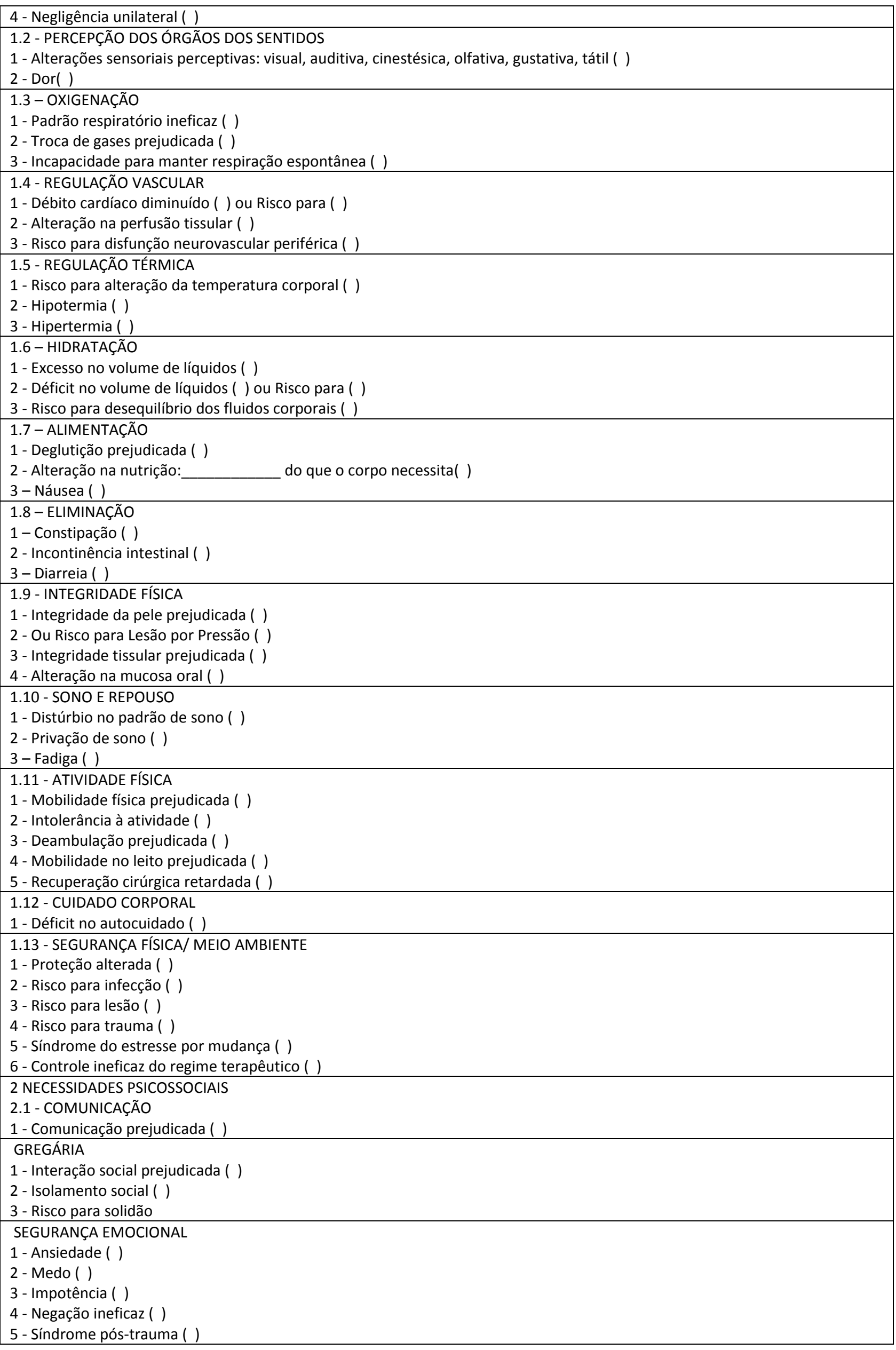




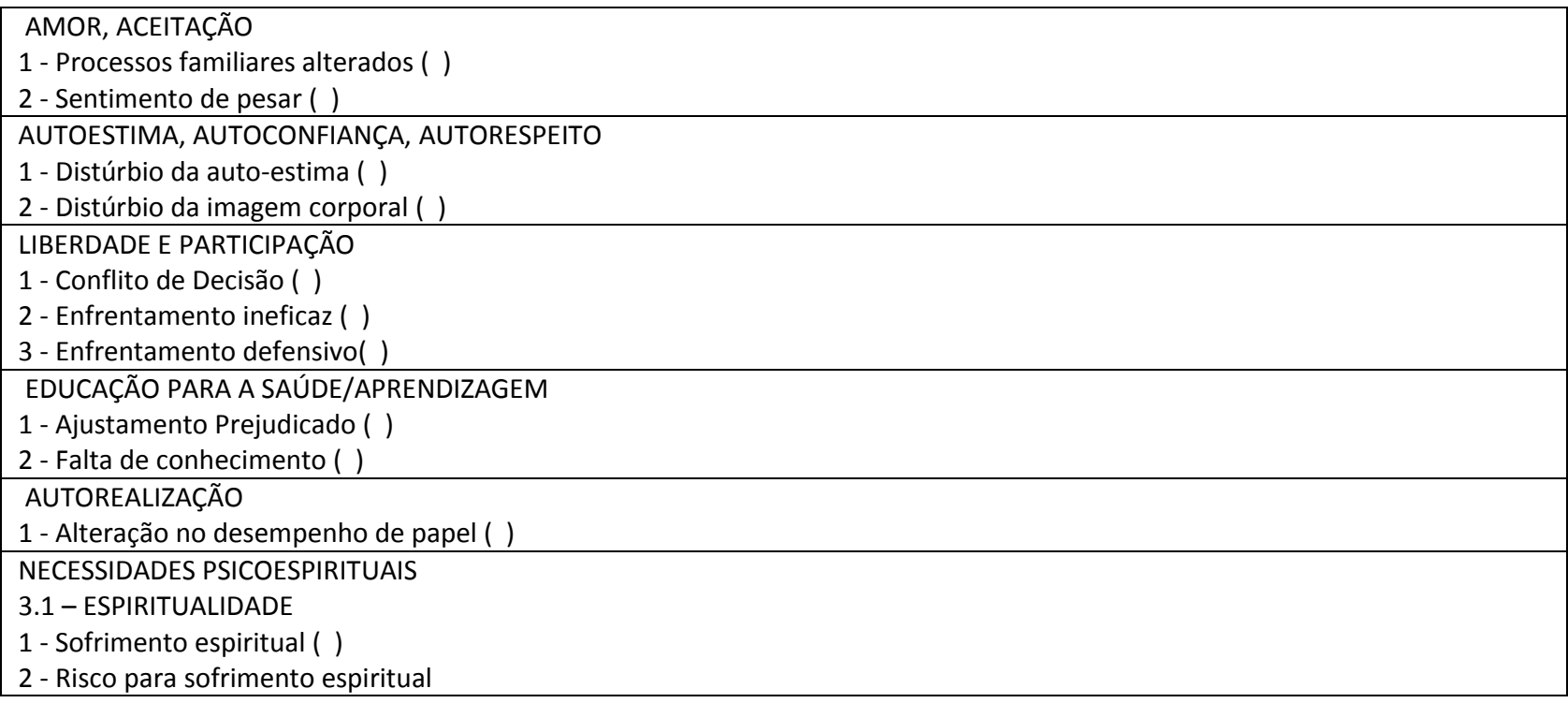

Os diagnósticos de enfermagem contemplam as NHB conforme sua divisão em: Necessidades Psicobiológicas, Necessidades Psicossociais e Necessidades Psicoespirituais, sendo que as necessidades psicobiológicas agregam a oxigenação, hidratação, eliminação, sono e repouso, nutrição, atividades físicas, motilidade, sexualidade, cuidado corporal, integridade cutâneo-mucosa e física, regulação térmica, neurológica, hidroeletrolítica, vascular, percepção dos órgãos do sentido, ambiente, terapêutica e locomoção. As necessidades psicossociais englobam segurança, amor e aceitação, liberdade e participação, comunicação, educação para saúde, gregária, autoestima, autoimagem e segurança emocional. As necessidades psicoespirituais contemplam as religiosas, éticas e de filosofia de vida ${ }^{(11-12)}$. Assim, 0 instrumento engloba praticamente todas as NHB que podem ser afetadas, fornecendo subsídios para o profissional enfermeiro traçar o plano de cuidados para o paciente ${ }^{(3)}$.

$O$ instrumento foi elaborado desta forma a fim de facilitar a identificação dos diagnósticos de enfermagem de acordo com as NHB afetadas que o paciente possa apresentar, pois a teoria funciona como um alicerce estrutural para a implantação da $\mathrm{SAE}$, que requer uma metodologia para ser implantada ${ }^{(12)}$. E, cabe ressaltar que os problemas de enfermagem encontrados na teoria das NHB relacionam-se com as características definidoras e fatores relacionados da NANDA (North American Nursing Diagnosis Association) $^{(11)}$.

Assim, o enfermeiro, a partir dos dados coletados no histórico de enfermagem e exame físico, deverá identificar os problemas de enfermagem e NHB afetadas e fará um julgamento clínico sobre as respostas do indivíduo, da família e comunidade aos problemas/processos de vida vigentes ou potenciais $^{(9)}$.

Embasado nos problemas e diagnósticos de enfermagem, o enfermeiro deve elaborar a prescrição de cuidados que é implementada pela equipe de enfermagem, oferecendo a assistência de que o paciente necessita ${ }^{(11)}$.

Desta maneira, a terceira parte do instrumento contempla a Prescrição de Enfermagem e evolução de enfermagem, conforme a Figura 3.

Figura 3 - Prescrição de enfermagem e evolução de enfermagem.

\begin{tabular}{|l|l|l|}
\hline \multicolumn{3}{|c|}{ PRESCRIÇÃO DE ENFERMAGEM } \\
\hline Nome: Clínica: _ LESPONSÁVEL \\
\hline PRESCRIÇÃO & APRAZAMENTO Leito: & \\
\hline & & \\
\hline & & \\
\hline & & \\
\hline & & \\
\hline
\end{tabular}




\section{DATA/HORA}

As prescrições de enfermagem são medidas para a solução dos diagnósticos de enfermagem identificados, registrados previamente pelo enfermeiro a partir da análise do histórico de enfermagem e exame físico. Devem ser avaliadas constantemente a fim de saber se estão sendo positivas para responder aos diagnósticos de enfermagem e a recuperação e manutenção ou não das NHB afetadas. Caso negativo, é responsabilidade do enfermeiro reavaliar sua conduta, fazer nova prescrição, pois a prescrição de enfermagem são medidas deliberadas por este profissional, que direciona e coordena a assistência de enfermagem ao paciente de forma individualizada e contínua, objetivando a prevenção, promoção, proteção, recuperação e manutenção da saúde ${ }^{(9-12)}$.

A evolução de enfermagem permite ao enfermeiro avaliar os resultados dos cuidados subsidiados na prescrição de enfermagem, incluindo, excluindo ou modificando as intervenções, conforme as respostas do paciente ao cuidado prestado. A cada 24 horas, deve-se atualizar a evolução, avaliando se os problemas foram ou não resolvidos, se novos problemas surgiram ou se modificaram com o cuidado de enfermagem ${ }^{(10-11)}$.

Sintetizando, o instrumento elaborado para a SAE é composto por três partes sequenciadas, na qual a segunda e a terceira partes compõem uma ficha diária, sendo que, a cada 24 horas, deverá ser realizada uma evolução a fim de analisar se os problemas de enfermagem foram ou não sanados. Cabe ressaltar que a implementação deste instrumento na rotina de enfermagem da clínica cirúrgica é um grande desafio devido haver algumas limitações que, no entanto, podem ser superadas com a organização laboral e administrativo.

Após a elaboração e discussão do instrumento em questão, ressalta-se a importância deste instrumento para a prática do enfermeiro durante sua assistência ao paciente, uma vez que o hospital para o qual este instrumento foi elaborado não conta com a SAE em seu cotidiano de atendimento. Neste sentido, o instrumento possibilitará implementar a sistematização da assistência de enfermagem na clínica cirúrgica do hospital, promovendo uma nova rotina quanto à utilização do impresso, oferecendo subsídios na qualidade da assistência de enfermagem e fortalecerá e valorizará as condutas da equipe de enfermagem e a avaliação e evolução do paciente pelo enfermeiro.

Desta forma, com este instrumento, buscase a melhoria da Sistematização da Assistência de Enfermagem no referido hospital, pois contempla todas as fases da sistematização, facilitando sua implementação na clínica por se tratar de um instrumento autoexplicativo da SAE. Dessa maneira, elaborar e discutir o instrumento durante o módulo, como método avaliativo, foi de grande relevância para o aperfeiçoamento profissional, pois a oportunidade de apresentar um instrumento já utilizado em outros hospitais e adequá-lo à realidade local foi gratificante e poderá subsidiar o trabalho de toda a equipe de enfermagem, permitindo a avaliação e evolução diária do paciente.

\section{CONCLUSÃO}

O instrumento elaborado é uma importante estratégia para a organização das ações e operacionalização do processo de enfermagem, pois padronizam os registros e respaldam legalmente as ações de enfermagem, além de permitir a continuidade do planejamento dos cuidados prestados.

Para o hospital, esse instrumento, se implantado, trará um ganho enorme para a enfermagem, pois a SAE ainda não é utilizada rotineiramente pela equipe. Dessa forma, o instrumento auxiliará na organização do trabalho e propiciará uma assistência sistematizada para o paciente, promovendo a autonomia do enfermeiro e unificando a linguagem com a equipe.

Desse modo, a contribuição deste relato de experiência foi para elaboração do instrumento conforme consta no trabalho a fim de colaborar significativamente com a equipe de enfermagem em sua sistematização da assistência ao paciente, ressaltando a necessidade de ampliar e aprofundar, continuamente, os saberes 
específicos de sua área de atuação, sem esquecer o enfoque interdisciplinar e assistência ao paciente.

\section{REFERÊNCIAS}

1. Paiano LAG, Matos FGOA, Richetti MAA, Casarolli ACG, Girardello DTF, Barbosa HBB, et al. Padronização das ações de enfermagem prescritas para pacientes clínicos e cirúrgicos em um hospital universitário. Rev Enferm Cent 0 Min. $2014 \quad$ set-dez;3(4):1336-48. http://dx.doi.org/10.19175/recom.v0i0.557

2. Paiva ACPC, Arreguy-Sena C, Alves MS, Salimena AMO. Construção de instrumentos para o cuidado sistematizado da enfermagem: mulheres em processo cirúrgico de mastectomia. Rev Enferm Cent O Min. 2016 mai-ago;6(2): 2282-91.

http://dx.doi.org/10.19175/recom.v6i2.707

3. Schmitz EL, Gelbcke FL, Bruggmann MS, Luz SCL. Filosofia e marco conceitual: estruturando coletivamente a sistematização da assistência de enfermagem. Rev Gaúcha Enferm. 2016;37(n. esp):1-9. $\quad$ http://dx.doi.org/10.1590/19831447.2016.esp.68435

4. Conselho Federal de Enfermagem (COFEN). Resolução no 358, de 15 de outubro de 2009. Dispõe sobre Sistematização da assistência de enfermagem (SAE) nas instituições de saúde brasileiras [Internet]. Brasília, DF; 2009 [citado em 2017 out 15]. Disponível em: http://www.cofen.gov.br/resoluo-cofen-

\section{4384.html}

5. Diagnósticos de Enfermagem da NANDA: definições e classificação 2015/2017. 10ạ ed. São Paulo: Artmed; 2015.

6. Santos N, Veiga P, Andrade R. Importância da anamnese e do exame físico para o cuidado do enfermeiro. Rev Bras Enferm. 2011 marabr;64(2):355-8.

http://dx.doi.org/10.1590/S0034-

\section{1}

7. Gutiérrez MGR, Morais SCRV. Sistematização da assistência de enfermagem e a formação da identidade profissional. Rev Bras Enferm. 2017 mar-abr;70(2):455-60.

http://dx.doi.org/10.1590/0034-7167-2016-0515

8. Souza TL, Trindade TRO, Mendonça AEO, Silva RAR. Necessidades humanas básicas alteradas em pacientes pós-transplante renal: estudo transversal. Braz J Nurs [Internet]. 2016 jun [citado em 2017 out 10];15(2):265-75. Disponível em:

\section{http://www.objnursing.uffbr/index.php/nursing/} article/view/5253

9. Oliveira RS, Almeida EC, Azevedo NM, Almeida MAP, Oliveira JGC. Reflexões sobre as bases científicas e fundamentação legal para aplicação da sistematização do cuidado de enfermagem. Rev UNIABEU [Internet]. 2015 set-dez [citado em 2017 set 10];8(20):350-62. Disponível em: http://revista.uniabeu.edu.br/index.php/RU/artic le/view/1912/pdf 298

10 Santos DMA, Sousa FGM, Paiva MVS, Santos AT, Pinheiro JMS. A enfermagem baseada em evidências apoiando a construção do histórico de enfermagem: uma pesquisa bibliográfica. Cienc Cuid Saude. 2016 jul-set;15(3):561-9. http://dx.doi.org/10.4025/cienccuidsaude.v15i3.2 $\underline{6357}$

11. Ubaldo I, Matos E, Salum NC. Diagnósticos de enfermagem da Nanda-I com base nos problemas segundo teoria de Wanda Horta. Cogitare Enferm [Internet]. 2015 out-dez [citado em 2017 nov 10];20(4):687-94. Disponível em: http://www.redalyc.org/articulo.oa?id=48364768 $\underline{1006}$

12. Remizoski J, Rocha MM, Vall J. Dificuldades na implantação da sistematização da assistência de enfermagem - SAE: uma revisão teórica. Cad Esc Saúde [Internet]. 2010 [citado em 2017 nov 20];3:1-14. Disponível em: http://revistas.unibrasil.com.br/cadernossaude/i ndex.php/saude/article/view/68/68

Nota: Este relato de experiência faz parte da monografia de final de curso da especialização na modalidade de Residência em Enfermagem Cirúrgica.

Recebido em: 27/05/2017

Aprovado em: 12/04/2018

Endereço de correspondência:

Fernanda Maryneve Menezes Tavares

Av. Fab, 69 - Centro

CEP: 68.900-000 - Macapá/ AP - Brasil

E- mail: fernanda maryneve@hotmail.com 